\title{
Impedance and Noise Closed-Form Model of Large-Area Integrated Resistors With High Stray Capacitance to be Used as Feedback Discharge Devices in Charge-Sensitive Preamplifiers for Nuclear Spectroscopy
}

\author{
Stefano Capra
}

\begin{abstract}
In this work a theoretical study and experimental measurements have been conducted in order to describe both the impedance and the noise associated with resistors with appreciable distributed stray capacitance. In particular the effects of using such devices as feedback discharge for nuclear spectroscopy charge-sensitive preamplifier have been analyzed. A closed-form model shows that for frequencies higher than $1 /(2 \pi \cdot R C / 2)$ the generated noise is not white anymore and the power spectral density of noise raises with the square root of frequency. The experimental measurements confirm the model validity.
\end{abstract}

\section{INTRODUCTION}

The main focus of this work is an accurate impedance and noise description of integrated, large-area, high-valued resistors with strong stray capacitance to bulk. Their use as feedback discharge devices for charge-sensitive preamplifiers will be discussed, with particular emphasis on the effects of the distributed stray capacitance on the power spectral density of noise. Such effects, under certain realistic conditions of total resistance and capacitance, can "colour" the noise even at low frequencies, down to some hundreds of $\mathrm{Hz}$.

When describing the most relevant noise sources in a charge-sensitive preamplifier for nuclear spectroscopy, those that are generally taken into account are the operational amplifier's input FET and the feedback resistor. The first one is responsible for three contributions:

- Series Johnson noise $4 k T \gamma / g_{m}$ proportional to the FET transconductance $g_{m}$ ( $\gamma$ is a non-dimensional parameter between $2 / 3$ and 2). This is the thermal noise of the channel.

- Parallel shot noise $2 q I_{G}$ due to gate current $I_{G}$, almost absent in CMOS transistors

- Series $1 / \mathrm{f}$ pink noise $k /\left(f C_{O X} W L\right)$ inversely proportional to the MOS channel dimensions $\mathrm{W}$ and L. This noise is caused by trapping-detrapping phenomena of charge carriers in stray energy levels on the interface between channel and oxide. $k$ is a device-specific parameter while $C_{O X}$ is the oxide dielectric coefficient.

S. Capra is with the Department of Physics, University of Milano and INFN of Milano, Italy (email: stefano.capra@unimi.it).

Manuscript received October 2019; revised February 2020.
The feedback resistor is generally considered to be responsible for a parallel white Johnson noise with physical spectral density equal to $4 k T / R$. To give a quantitative example, a $1 \mathrm{G} \Omega$ and a $100 \mathrm{M} \Omega$ resistors generate respectively a power spectral density of noise equal to $16.6 \times 10^{-30} \frac{\mathrm{A}^{2}}{\mathrm{~Hz}}$ and $16.6 \times 10^{-29} \frac{\mathrm{A}^{2}}{\mathrm{~Hz}}$. The feedback resistor's value is obviously maximized in order to obtain a better spectroscopic resolution, since its noise sums up directly to the one generated by the detector. A 1 or $2 \mathrm{G} \Omega$ resistor is generally suitable for the requirements of $\gamma$ spectroscopy. In case of X-spectroscopy the required resistance value can rise and even unconventional $1 \mathrm{~T} \Omega$ resistors may be used. In conventional discrete-type circuits these are thinfilm metal resistors, but the progressive integration of the front-end circuits into ASICs requires, in most cases, the integration of the feedback devices too. As will be pointed out throughout this work, the integration of a $100 \mathrm{M} \Omega$ or higher resistor is definitely not a trivial task. However in some applications this may become a preferable solution. For example, active transconductors generally aren't compliant with the requirements of high-resolution gamma spectroscopy due to their low linearity: in order to achieve the highest resolution possible, moving window deconvolution algorithms require the preamplifier signals to have a pure exponential shape that is not obtainable using, for example, a MOS transistor in triode region [1]. Some solutions based on lownoise CMOS structures that can substitute a real resistor are under study [2].

SMD (Surface Mounting Devices) resistors are known to have a relatively high density of radioactive contaminants in their ceramic substrate. Those experiments looking for rare decays and usually taking place in underground laboratories require an ultra-low radiation background. In such experimental contexts high-value polysilicon resistors in bare-die format are an interesting alternative to SMD resistors as feedback devices for discrete-type charge-sensitive preamplifiers [3]. In fact silicon wafers are known to be naturally radio-purer than ceramic materials.

Contemporary technologies suitable for analog designs often include a high-resistivity module that allow for the realization of polysilicon resistors. Such polysilicon layer has 
generally a resistivity in the order of $1 \mathrm{k} \Omega / \square$. Considering a minimum polysilicon width of $1 \mu \mathrm{m}$, a $1 \mathrm{G} \Omega$ resistor would occupy roughly $1 \mathrm{~mm}^{2}$, and this not considering the required empty space to allow the resistance to fold without shortcircuiting its segments.

The huge area consumption of such devices, however, is not their only drawback: they have a huge capacitance to bulk. For example, a $100 \mathrm{M} \Omega$ resistor realized in AMS $350 \mathrm{~nm}$ [4] shows a total stray capacitance to bulk in the order of $10 \mathrm{pF}$. This capacitance turns the resistor into some sort of transmission line that is far from the ideal resistor model both in terms of impedance and noise. In the next sections a closed-form exact calculation of both these quantities is reported. Experimental measurements conducted with an integrated resistor and a lownoise charge-sensitive preamplifier confirm the validity of the proposed model.

\section{IMPEDANCE CALCULATION OF A RESISTOR WITH DISTRIBUTED CAPACITANCE}

The closed-form impedance description of a resistor with distributed capacitance (we will refer to this kind of device throughout the text with the acronym " $R W D C$ ") is not trivial. Some works on the topic are available in literature [5] with different degrees of approximation. In this section a closedform didactic approach to the description of such devices is presented. Please notice that throughout the text $s=i \omega$. In the same way the voltages $V_{0}, V_{1}$ and the currents $I_{0}, I_{1}$ should be intended as functions of the complex frequency $s$.

In order to calculate its behaviour on the s-plane we start from a simple lumped-parameter R-C cell (see Fig. 1).
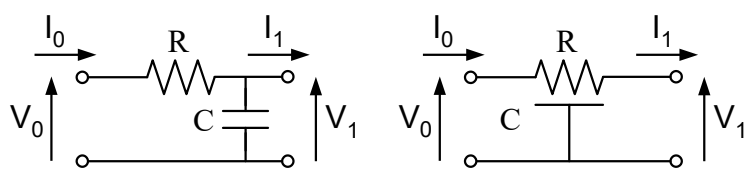

Fig. 1. Left: simple lumped-parameter resistance with capacitive coupling to bulk. Right: distributed-parameter resistance of value $\mathrm{R}$ with total capacitive coupling C.

Such elementary circuit obeys to the following equation:

$$
\left[\begin{array}{l}
V_{0} \\
V_{1}
\end{array}\right]=\left[\begin{array}{cc}
R+\frac{1}{s C} & -\frac{1}{s C} \\
\frac{1}{s C} & -\frac{1}{s C}
\end{array}\right] \cdot\left[\begin{array}{l}
I_{0} \\
I_{1}
\end{array}\right] \text {. }
$$

We now rewrite (1) in order to better suit our purposes.

$$
\left[\begin{array}{c}
V_{0} \\
I_{0}
\end{array}\right]=\left[\begin{array}{cc}
1+s R C & R \\
s C & 1
\end{array}\right] \cdot\left[\begin{array}{l}
V_{1} \\
I_{1}
\end{array}\right]
$$

We will use this formulation because it allows to study chains of double bipoles with simple matrix multiplications. More specifically, the output current $I_{1}$ and voltage $V_{1}$ of the previous cell are equal to the input current $I_{0}$ and voltage $V_{0}$ of the following one. We will now chain together $\mathrm{n}$ R-C cells as seen in Fig. 1, each one with a resistance and capacitance equal to $R / n$ and $C / n$. In this way we obtain the circuit in Fig. 2.

The equation that describes such system is the following:

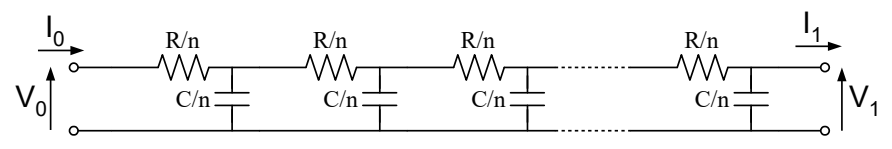

Fig. 2. First-order approximation of a distributed resistance $\mathrm{R}$ with capacitive coupling C made of a series of n R-C cells with resistance $\frac{R}{N}$ and capacitance $\frac{C}{N}$.

$$
\left[\begin{array}{c}
V_{0} \\
I_{0}
\end{array}\right]=\left[\begin{array}{cc}
1+s \frac{R C}{n^{2}} & \frac{R}{n} \\
\frac{s C}{n} & 1
\end{array}\right]^{n} \cdot\left[\begin{array}{l}
V_{1} \\
I_{1}
\end{array}\right]
$$

Most computer simulators implement nothing but this model for integrated RWDCs. As we will calculate soon, this approximation may be too coarse or acceptable depending on the RWDC segmentation. In order to proceed with the calculus we need to diagonalize the matrix in (3). The eigenvalues $\lambda_{1,2}$ and eigenvectors $v_{1,2}$ are reported respectively in (4) and (5).

$$
\begin{gathered}
\lambda_{1,2}=\frac{\mp \sqrt{s R C\left(s R C+4 n^{2}\right)}+s R C+2 n^{2}}{2 n^{2}} \\
v_{1,2}=\left[\begin{array}{c}
\frac{R \sqrt{s C} \mp \sqrt{R\left(4 n^{2}+s R C\right)}}{2 n \sqrt{s C}} \\
1
\end{array}\right]
\end{gathered}
$$

We are now able to rewrite (3) in a more interesting form:

$$
\left[\begin{array}{c}
V_{0} \\
I_{0}
\end{array}\right]=[B] \cdot\left[\begin{array}{cc}
\lambda_{1}^{n} & 0 \\
0 & \lambda_{2}^{n}
\end{array}\right] \cdot\left[B^{-1}\right] \cdot\left[\begin{array}{c}
V_{1} \\
I_{1}
\end{array}\right]
$$

where the two matrices $B$ and $B^{-1}$ are respectively

$$
B=\left[\begin{array}{cc}
\frac{R \sqrt{s C}-\sqrt{R\left(4 n^{2}+s R C\right)}}{2 n \sqrt{s C}} & \frac{R \sqrt{s C}+\sqrt{R\left(4 n^{2}+s R C\right)}}{2 n \sqrt{s C}} \\
1 & 1
\end{array}\right]
$$

and

$$
B^{-1}=\left[\begin{array}{cc}
-\frac{n \sqrt{s C}}{\sqrt{R\left(4 n^{2}+s R C\right)}} & \frac{R \sqrt{s C}+\sqrt{R\left(4 n^{2}+s R C\right)}}{2 \sqrt{R\left(4 n^{2}+s R C\right)}} \\
\frac{n \sqrt{s C}}{\sqrt{R\left(4 n^{2}+s R C\right)}} & \frac{-R \sqrt{s C}+\sqrt{R\left(4 n^{2}+s R C\right)}}{2 \sqrt{R\left(4 n^{2}+s R C\right)}}
\end{array}\right] .
$$

In order to switch from an approximated segmented RWDC to a closed-form continuous model we should just evaluate (6) in the limit for $n \rightarrow \infty$. We can evaluate the limit of $B, B^{-1}$ and the eigenvalues separately: their analytical expressions converge. The result is the following:

$$
\left[\begin{array}{l}
V_{0} \\
I_{0}
\end{array}\right]=\left[\begin{array}{cc}
-\sqrt{\frac{R}{s C}} & \sqrt{\frac{R}{s C}} \\
1 & 1
\end{array}\right] \cdot\left[\begin{array}{cc}
e^{-\sqrt{s R C}} & 0 \\
0 & e^{\sqrt{s R C}}
\end{array}\right] \cdot\left[\begin{array}{cc}
-\frac{\sqrt{s C}}{2 \sqrt{R}} & \frac{1}{2} \\
\frac{\sqrt{s C}}{2 \sqrt{R}} & \frac{1}{2}
\end{array}\right] \cdot\left[\begin{array}{l}
V_{1} \\
I_{1}
\end{array}\right]
$$


Using trigonometric transformations, the final expression of 9 becomes:

$$
\left[\begin{array}{c}
V_{0} \\
I_{0}
\end{array}\right]=\left[\begin{array}{cc}
\cosh (\sqrt{s R C}) & \sqrt{\frac{R}{s C}} \sinh (\sqrt{s R C}) \\
\sqrt{\frac{s C}{R}} \sinh (\sqrt{s R C}) & \cosh (\sqrt{s R C})
\end{array}\right] \cdot\left[\begin{array}{c}
V_{1} \\
I_{1}
\end{array}\right] .
$$

The impedance expression of the resistance with distributed capacitance is implicitly contained in (10). The impedance matrix can be obtained rearranging the terms so as to obtain a voltage-only vector on the left and a current-only vector on the right. The impedance $Z$ matrix thus is equal to:

$$
Z_{R W D C}=\sqrt{\frac{R}{s C}} \cdot\left[\begin{array}{cc}
\operatorname{coth}(\sqrt{s R C}) & -\frac{1}{\sinh (\sqrt{s R C})} \\
\frac{1}{\sinh (\sqrt{s R C})} & -\operatorname{coth}(\sqrt{s R C})
\end{array}\right]
$$

where

$$
\left[\begin{array}{l}
V_{0} \\
V_{1}
\end{array}\right]=\left[Z_{R W D C}\right] \cdot\left[\begin{array}{c}
I_{0} \\
I_{1}
\end{array}\right]
$$

It is straightforward to see that, for $C \rightarrow 0$, (10) assumes the trivial expression in (13).

$$
\left[\begin{array}{c}
V_{0} \\
I_{0}
\end{array}\right]=\left[\begin{array}{cc}
1 & R \\
0 & 1
\end{array}\right] \cdot\left[\begin{array}{l}
V_{1} \\
I_{1}
\end{array}\right]
$$

This result guarantees the coherence of the proposed model in the limit of no capacitive coupling with the behaviour of an ideal resistor.

\section{USING A RWDC AS FEEDBACK RESISTOR IN A CHARGE-SENSITIVE PREAMPLIFIER}

Given the results pointed out in the previous section let's now analyze the effects of the non-trivial RWDC impedance when using it as feedback resistor of a Charge-Sensitive Preamplifier (CSP) (see Fig. 3). Please notice that in this section we refer to the feedback capacitance with $C_{F}$ and to the RWDC stray capacitance with $C$.

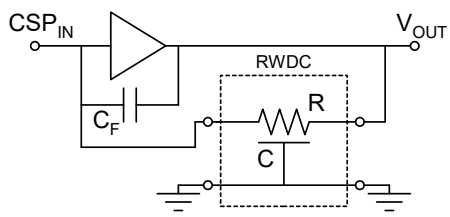

Fig. 3. Conceptual schematic of a CSP with an RWDC used as feedback resistor.

The equation in (10) can be rewritten considering that $V_{1}=$ $V_{\text {OUT }}$ is the output voltage of the CSP and $V_{0}=0$ because the CSP input node is virtual ground. The feedback complex impedance can be expressed as:

$$
Z_{R W D C}=-\frac{V_{1}}{I_{0}}=\sqrt{\frac{R}{s C}} \sinh (\sqrt{s R C}) .
$$

From this equation we can derive

$$
\frac{V_{\text {OUT }}}{i_{I N}}(s)=-\left(\frac{1}{s C}\right) \frac{\sqrt{s R C} \sinh (\sqrt{s R C})}{1+\left(\frac{C_{F}}{C}\right) \sqrt{s R C} \sinh (\sqrt{s R C})}
$$

that is the expression of the CSP's transfer function between input current $i_{I N}$ and output voltage. It is possible to perform the Puiseux expansion [6], [7] of the hyperbolic sine in (15) for $s=0$ and truncate it after the second term, obtaining the following result:

$$
\frac{V_{O U T}}{i_{I N}}(s)=-R \frac{1+s \frac{R C}{6}}{1+s C_{F} R+s^{2} \frac{R^{2} C C_{F}}{6}} .
$$

This expression has one zero in

$$
s_{Z E R O}=-\frac{6}{R C}
$$

and two poles in

$$
s_{P O L E S}=-\frac{3}{R C}\left[1 \pm \sqrt{1-\frac{2 C}{3 C_{F}}}\right] .
$$

Despite this approximation comes from the truncation of the Puiseux series of the hyperbolic sine around $s=0$, the validity of the approximation extends way above the limit of low frequencies. In fact for $s \gg 1 / R C$ both the original and the approximated functions behave like $1 / s R C_{F}$. In practice the approximation effects are appreciable only around the frequency of the zero in (17) where the functions show a resonant behaviour. It should also be also pointed out that if the second-order truncation is a good approximation of the function, the fourth-order one is practically indistinguishable from the original (see Fig. 4).

In order to study the behaviour of (16) we split the discussion in two separate limit cases: $C<<C_{F}$ and $C>>C_{F}$.

\section{A. Low-parasitic-capacitance case: $C<<C_{F}$}

If the total RWDC stray capacitance is considerably lower than the feedback one, we can expand the square root of the expression in (18) in terms of $C / C_{F}$. The formulas of the two poles can now be written as:

$$
S_{P O L E 1}=-\frac{1}{R C_{F}}
$$

and, with $\alpha=C /\left(6 C_{F}\right)$,

$$
s_{P O L E 2}=-\frac{6}{R C}(1-\alpha) .
$$

When $\alpha$ goes to 0 , i.e. it is negligible respect to the feedback capacitance, the zero in (17) and the pole in (20) tend to cancel out reciprocally, giving a conventional CSP transfer function dominated by the pole in (19).

If the RWDC stray capacitance is not completely negligible respect to the feedback capacitance, the second pole moves away (on the real axis) from the zero. Using the expression of the poles in (19) and (20) the CSP transfer function becomes:

$$
\frac{V_{\text {OUT }}}{i_{I N}}(s)=-R \cdot \frac{1+s \frac{R C}{6}}{\left(1+s R C_{F}\right)\left(1+s \frac{R C}{6(1-\alpha)}\right)(1-\alpha)}
$$



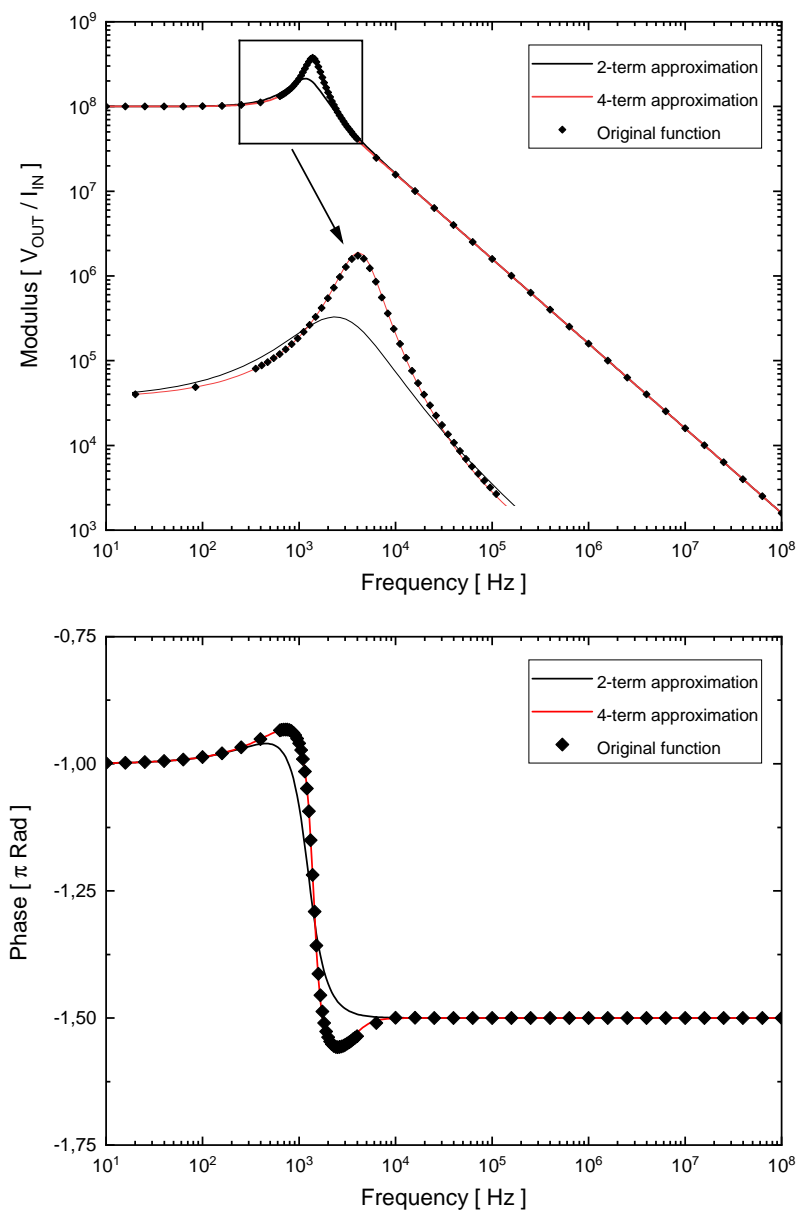

Fig. 4. Comparison between the magnitude and phase of the preamplifier response in (15) and the corresponding simplification in (16). For completeness, a fourth-order approximation is also added. In this example $\mathrm{R}=100 \mathrm{M} \Omega$, $\mathrm{C}=10 \mathrm{pF}, \mathrm{C}_{F}=1 \mathrm{pF}$.

where the term $(1-\alpha)$ is introduced to renormalize the highfrequency limit of the denominator so that it coincides with the one in (16). With no approximations (21) can be rewritten as

$$
\frac{V_{O U T}}{i_{I N}}(s)=-\frac{R}{1+s R C_{F}}-\frac{R}{1+s R C_{F}} \cdot \frac{\left(\frac{\alpha}{1-\alpha}\right)}{1+s R C_{F}\left(\frac{\alpha}{1-\alpha}\right)} .
$$

We can easily calculate the inverse Laplace transform of the expression in (22) pointing out some considerations. The first term represents the typical exponential decay of a chargesensitive preamplifier. The second term is a two-pole function that corresponds in the time domain to the convolution of two exponentials. The impulse-response function can be written as

$$
V_{\text {OUT }}=-\frac{\theta(t)}{C_{F}} e^{-\frac{t}{R C_{F}}}-\frac{\theta(t)}{C_{F}} e^{-\frac{t}{R C_{F}}} * \frac{\theta(t)}{R C_{F}} e^{-\frac{t}{\left(\frac{\alpha}{1-\alpha}\right) R C_{F}}} .
$$

Calculating the convolution and writing $\beta=\alpha /(1-2 \alpha)$ we obtain the unitary CSP impulse response function:

$$
V_{\text {OUT }}(t)=-\frac{\theta(t)}{C_{F}} e^{-\frac{t}{R C_{F}}}\left[1+\beta\left(1-e^{-\frac{t}{\beta R C_{F}}}\right)\right] .
$$

The derivative of this function is zero for $t \rightarrow 0^{+}$. A simple interpretation of (24) is that the CSP impulse response function is an exponential decay signal that starts with a flat tip. The characteristic time of this deviation from ideality is $\beta R C_{F}$.

\section{B. High-parasitic-capacitance case: $C>>C_{F}$}

In case of high parasitic capacitance the argument of the square root in the poles expression (18) is negative and this creates two complex-conjugate splitted poles:

$$
s_{P O L E(1,2)}=-\frac{3}{R C}\left(1 \pm i \sqrt{\frac{2 C}{3 C_{F}}}\right)=-3 \frac{1 \pm i 2 \sqrt{\alpha}}{R C} .
$$

The CSP transfer function can thus be written like

$$
\frac{V_{O U T}}{i_{I N}}(s)=-R \cdot \frac{1+s \frac{R C}{6}}{\left(s+3 \frac{1+i 2 \sqrt{\alpha}}{R C}\right) \cdot\left(s+3 \frac{1-i 2 \sqrt{\alpha}}{R C}\right) \frac{R^{2} C C_{F}}{6}}
$$

where $R^{2} C C_{F} / 6$ is again the high-frequency normalization. Remembering that $\alpha=C /\left(6 C_{F}\right)$, the CSP impulse response function thus becomes

$$
V_{\text {OUT }}(t)=-\frac{1}{C_{F}} e^{-\frac{t}{2 \alpha R C_{F}}}\left[\cos \left(\frac{t}{\sqrt{\alpha} R C_{F}}\right)+\frac{1}{2 \sqrt{\alpha}} \sin \left(\frac{t}{\sqrt{\alpha} R C_{F}}\right)\right]
$$

that still has a zero derivative for $t \rightarrow 0^{+}$but has a damped oscillating behaviour due to the pole splitting.

\section{APPROXIMATION OF AN IDEAL RESISTOR WITH AN RWDC CHAIN}

The RWDC non-idealities come from the resistor parasitic coupling to the underlying surface. If we imagine to drive the potential of such surface so that it is equal point-to-point to the one of the overlying resistor we would completely cancel out the capacitive effects and the RWDC would become an ideal resistor again. This could be achieved realizing two superimposed resistors with the same shape but very different resistivity: the upper would be the high-value resistor and the lower would act as a low-impedance guard. Unfortunately this is not feasible due to common limitations of integrated technologies: for example it is not generally possible to stack two polysilicon layers with different resistivities.

On the other hand it is common to realize integrated polysilicon resistors with a n-well acting as a guard. As was previously pointed out in [8] and later in [9], despite the nonidealities described in the previous section, it is possible to obtain an approximated ideal-resistor behaviour from a chain of RWDCs. The core of this idea is to separate the RWDC in various segments, each one with its own underlying n-well driven with appropriate voltages so as to cancel out, in firstorder approximation, the capacitive effects.

Let's consider a RWDC realized on the top of a n-well and imagine to control the well voltage with an external circuit. Let's put this voltage equal to the mean of the voltages at the two resistor terminals. In order to do so, let's rewrite $V_{0}$ and $V_{1}$ in terms of $V_{D M}$ and $V_{C M}$ (see Fig. 5)

The equation in (10) can now be rewritten in terms of the newly introduced variables.

$\left[\begin{array}{cc}V_{C M}+\frac{V_{D M}}{2} \\ I_{0}\end{array}\right]=\left[\begin{array}{cc}\cosh (\sqrt{s R C}) & \sqrt{\frac{R}{s C}} \sinh (\sqrt{s R C}) \\ \sqrt{\frac{s C}{R}} \sinh (\sqrt{s R C}) & \cosh (\sqrt{s R C})\end{array}\right] \cdot\left[\begin{array}{c}V_{C M}-\frac{V_{D M}}{2} \\ I_{1}\end{array}\right]$. 

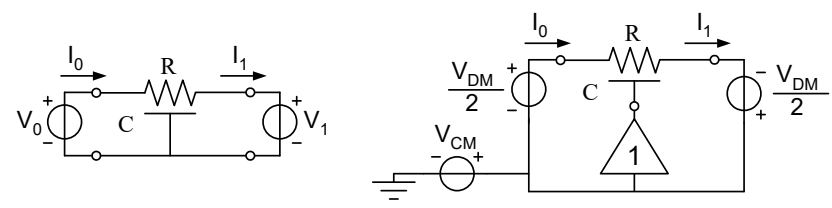

Fig. 5. Representation of the two different RWDC circuit descriptions using the variable sets $\left(V_{0}, V_{1}\right)$ and $\left(V_{D M}, V_{C M}\right)$.

Let's now put $V_{C M}=0$, since it doesn't affect the circuit anymore. From (10) and from symmetry considerations we easily obtain that $I_{0}=I_{1}$. The RWDC with auxiliary welldriving circuit can be considered as a simple passive dipole with complex impedance equal to

$$
Z_{e q}=\frac{V_{D M}}{I_{0}}=2 \sqrt{\frac{R}{s C}}\left[\tanh \left(\frac{1}{2} \sqrt{s R C}\right)\right] .
$$

Let's now imagine that we want to realize an integrated resistor with total resistance $R_{T O T}$. This device has a total stray capacitance equal to $C_{T O T}$. In order to minimize the capacitive coupling effects we subdivide this device in $n$ segments: each one is a RWDC with resistance $R_{T O T} / n$ and capacitance $C_{T O T} / n$ (see Fig. 6). Each of these is realized over a n-well. If $V_{1, m}$ and $V_{2, m}$ are the voltages at the two terminals of the $m$ th RWDC, the underlying $m$-th well is driven with a voltage equal to $\left(V_{1, m}+V_{2, m}\right) / 2$. Due to the simmetry of the system the wells can be driven by a simple low-impedance resistive voltage divider. Thanks to this solution the impedance of each of the $n$ segments is equal to

$$
Z_{S E G M E N T}=2 \sqrt{\frac{R_{T O T}}{s C_{T O T}}}\left[\tanh \left(\frac{1}{2} \sqrt{s \frac{R_{T O T}}{n} \frac{C_{T O T}}{n}}\right)\right] .
$$

A series of $n$ such RWDC, that has the desired total resistance equal to $n \cdot R_{T O T} / n=R_{T O T}$ has thus an impedance equal to:

$$
Z_{\text {CHAIN }}=2 n \sqrt{\frac{R_{T O T}}{s C_{T O T}}}\left[\tanh \left(\frac{1}{2} \sqrt{s \frac{R_{T O T}}{n} \frac{C_{T O T}}{n}}\right)\right] .
$$

This equation is telling us the following concept: given a total resistance, the higher the number of segments, the better the impedance approximation. In order to visualize the meaning of (31) the impedance of a RWDC chain with different number of segments is reported in Fig. 7.

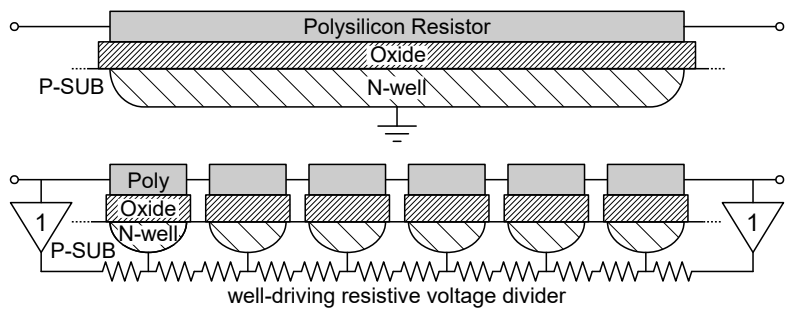

Fig. 6. Pictorial representation of the cross section of a of a polysilicon resistor realized over a n-well. Top figure: the resistor is realized over a single n-well guard. Bottom figure: the resistor is segmented into several parts, each one with its own n-well guard. Since the n-wells voltages follow the ones of the resistors, the capacitive effects are minimized.
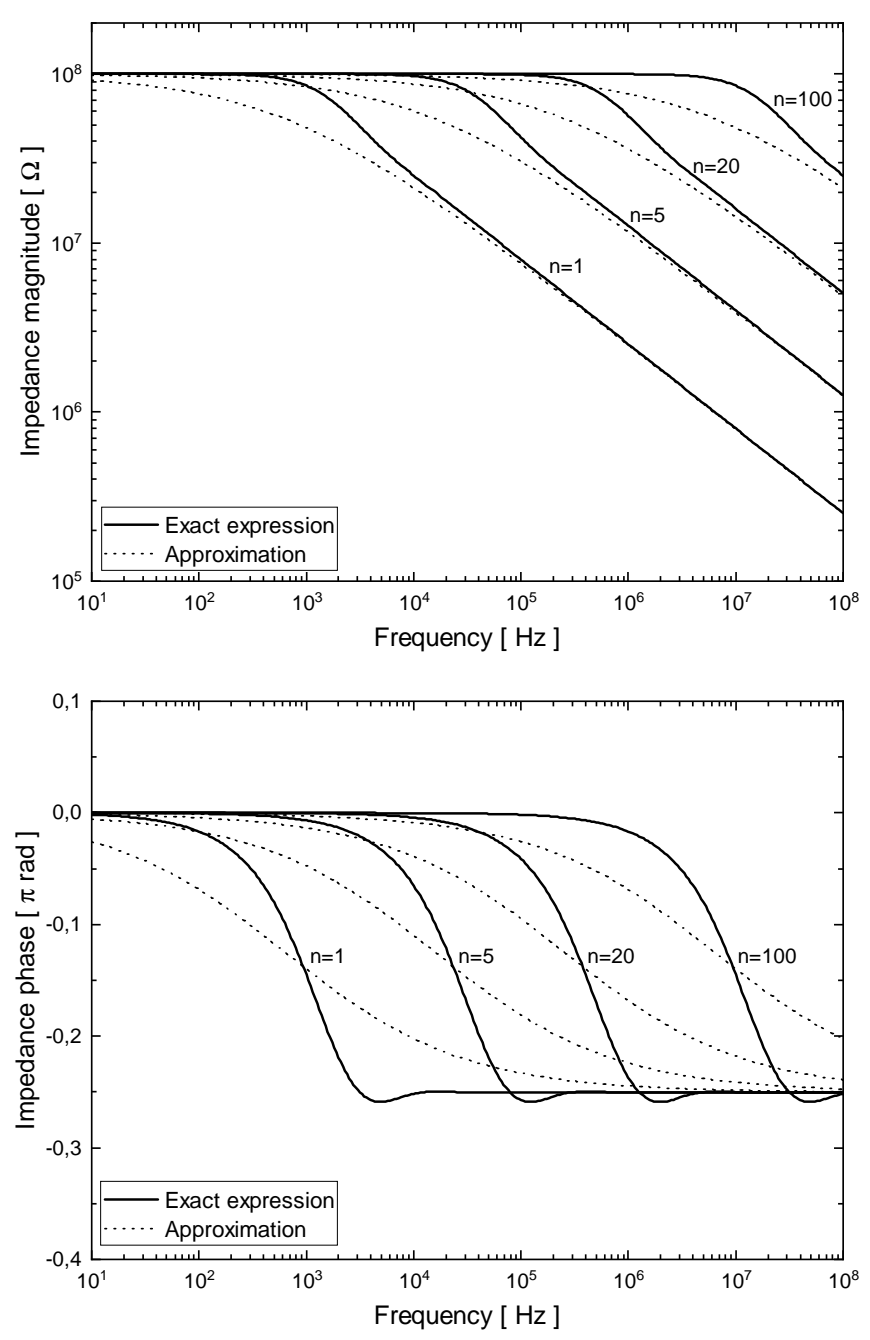

Fig. 7. Impedance (magnitude and phase) of a chain of RWDCs with the well-driving circuit. The number of segments is varied from $n=1$ to $n=100$. The total resistance is $100 \mathrm{M} \Omega$ and the total capacitance is $10 \mathrm{pF}$. The exact expression in (31) is compared to the approximation in (32).

We can approximate (31) considering its asymptotic behaviour at low and high frequency:

$$
Z_{C H A I N}=\frac{R}{1+\frac{1}{2 n} \sqrt{s R_{T O T} C_{T O T}}} .
$$

This result tells us that, in first approximation, the chain of RWDCs with well-driving circuits behaves like a real resistor up to a maximum frequency $f_{M A X}$ of:

$$
f_{M A X}=\frac{4 n^{2}}{2 \pi R_{T O T} C_{T O T}}=\frac{4 n^{2}}{2 \pi \gamma R^{2}} \quad \text { where } \quad \gamma=\frac{C_{T O T}}{R_{T O T}} .
$$

The latter expression gives a good indication about the number of required segments to obtain a good ideal-resistor emulation up to a specific frequency limit. Please notice that for each technology the $\gamma$ factor is fixed and describes the specific RWDC capacitance for unit resistance and depends on the polysilicon resistivity, the minimum resistor width, the thickness of the silicon oxide between the resistor and bulk and some geometrical layout parameters. 
When using a RWDC chain as feedback resistor in an integrated charge-sensitive amplifier (see Fig. 8) the implementation of the aforementioned well-driving circuit can be quite straightforward. Let the RWDC be segmented in an appropriate number of segments (according to the desired bandwidth). One node of the RWDC chain is to be connected to the CSP's input, i.e. virtual ground. The other terminal is driven by the low-impedance op-amp output stage. A passive, resistive voltage divider connected between the preamplifier output and ground can be satisfactorily used to drive the series of n-wells. This resistor chain must have a very low resistance compared to the RWDC. In this way its physical size will be much smaller than the main resistor and all the stray poles and zeros it introduces will lay well above the bandwidth of the circuit. As a rule of thumb we can ask that

$$
\frac{1}{2 \pi R_{D I V I D E R} C_{T O T}}>>f_{M A X} \text {. }
$$

The lower limit for the resistance value is essentially determined by the maximum power that can be dissipated by the CSP.

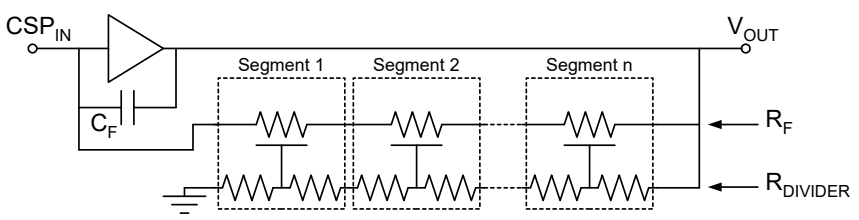

Fig. 8. Conceptual schematic of a charge-sensitive preamplifier with an RWDC used as feedback resistor $\left(R_{F}\right)$. In the picture are visible the feedback capacitance $C_{F}$, the high-resistivity polysilicon layer used as high-value feedback resistor $\left(R_{F}\right)$ and the low-resistivity passive voltage divider $R_{D I V I D E R}$ used to bias the n-wells underlying the RWDC.

In Fig. 9 the response function of a simple test-bench realization of the circuit in Fig. 8 is pictured. A commercial discrete op-amp was used together with an integrated polysilicon resistor (see next section). The difference in response shape is evident: when driving the n-wells with the voltage divider (in the $\mathrm{k} \Omega$ range) the signal has a clear exponential shape. On

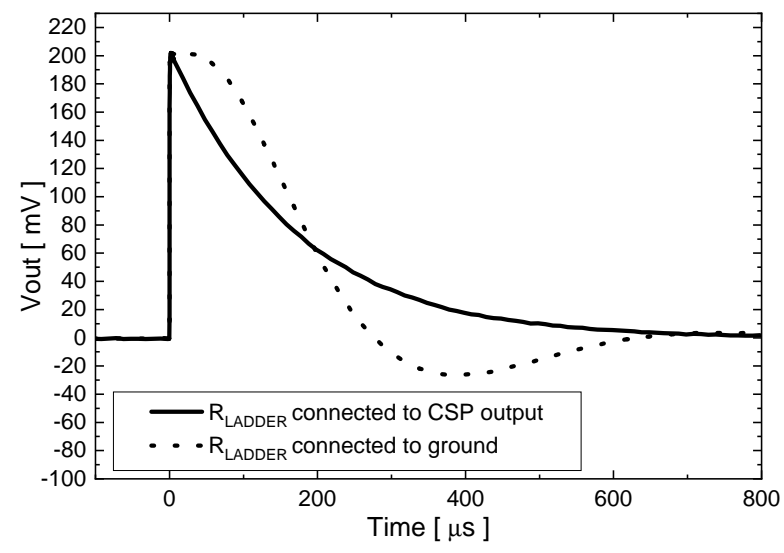

Fig. 9. Experimental impulse response of a CSP with RWDC feedback network (see Fig. 8). The two signals are obtained respectively biasing the n-wells with the resistive divider (solid line) or connecting them to ground (dashed line). For this test a commercial LM6171 operational amplifier was used. the other hand, keeping the wells to ground induces a peculiar transmission-line behaviour of the resistor as anticipated in (27).

\section{General NOISE MODEL OF A RWDC}

In a RWDC the generation of the noise is uniformly distributed across the polysilicon volume. The interaction of the noise sources with the distributed resistance and capacitance causes the total noise at the terminals, as expected, to not be white. In order to estimate the RWDC Power Spectral Density (PSD) of noise we should make an approximation: let's consider the device to be constituted by a series of $n$ RWDC segments, each one with a voltage noise generator with PSD equal to $4 k T R / n$ (see Fig. 10). Each noise generator is completely uncorrelated with the others and corresponds to the noise produced by a resistor with value $R / n$. The higher the number of segments, the better the approximation becomes. The result obtainable with a number of segments approaching infinite is the model of our continuous system.

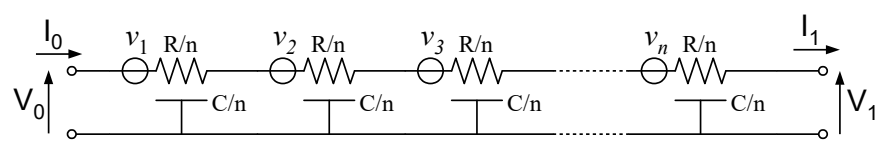

Fig. 10. Resistance with distributed capacitance modelled with a series of $n$ RWDC cells. Each one has resistance $\frac{R}{n}$ and capacitance $\frac{C}{n}$. A voltage noise generator $v_{n}$ with PSD of noise equal to $4 k T \frac{R}{n}$ is associated with each resistor.

The total noise power at the terminals is the sum of the power contributions from each noise generator. Let's consider, among a group of $n$ segments, the generic m-th noise generator: it has an rms value of $2 \sqrt{k T \frac{R}{n}}$ and is preceded by $m$ noisless RWDC cells and followed by $n-m$ noisless RWDC cells (see Fig. 11). At the moment let's treat it like a simple voltage generator. A series of identical RWDC segments is indistinguishable from a single RWDC with resistance and capacitance equal to the sum of the segments.

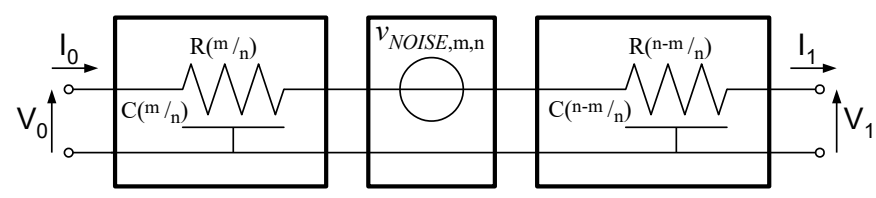

Fig. 11. Pictorial view of the circuit represented in (35), where the $m$-th noise generator is preceded by $m$ segments and followed by $n-m$.

In order to correctly describe the circuit in Fig. 11, we need to switch our formalism to an affine, 3-dimensional space [10].

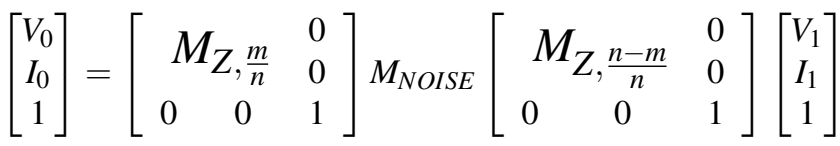




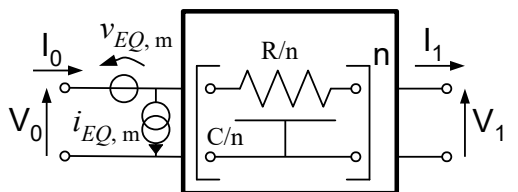

Fig. 12. Representation of the circuit in Fig. 11 substituting the voltage generator $v_{N O I S E, m}$ with the two equivalent generators $v_{E Q, m}$ and $i_{E Q, m}$. These generators are correlated and their respective sign is represented by the arrows.

where $M_{Z, k}$ is equal to

$$
\begin{aligned}
M_{Z, k} & =\left[\begin{array}{cc}
\cosh (k \sqrt{i \omega R C}) & \sqrt{\frac{R}{i \omega C}} \sinh (k \sqrt{i \omega R C}) \\
\sqrt{\frac{i \omega C}{R}} \sinh (k \sqrt{i \omega R C}) & \cosh (k \sqrt{i \omega R C})
\end{array}\right] \\
& =\left[\begin{array}{ll}
M_{Z, k, 1,1} & M_{Z, k, 1,2} \\
M_{Z, k, 2,1} & M_{Z, k, 2,2}
\end{array}\right]
\end{aligned}
$$

and $M_{N O I S E}$ is equal to

$$
M_{\text {NOISE }}=\left[\begin{array}{ccc}
1 & 0 & v_{N O I S E, m, n} \\
0 & 1 & 0 \\
0 & 0 & 1
\end{array}\right] .
$$

Considering that

$$
M_{Z, \frac{m}{n}} \cdot M_{Z, \frac{n-m}{n}}=M_{Z, 1}
$$

the equation in (35) can be rewritten as

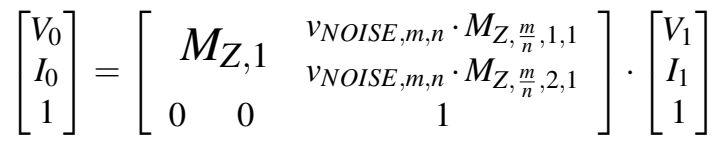

that is in turn equal to

$$
\begin{aligned}
& {\left[\begin{array}{c}
V_{0} \\
I_{0} \\
1
\end{array}\right]=} \\
& {\left[\begin{array}{ccc}
\cosh (\Omega) & \sqrt{\frac{R}{i \omega C}} \sinh (\Omega) & v_{N O I S E, m, n} \cdot \cosh \left(\frac{m}{n} \Omega\right) \\
\sqrt{\frac{i \omega C}{R}} \sinh (\Omega) & \cosh (\Omega) & v_{N O I S E, m, n} \cdot \sqrt{\frac{i \omega C}{R} \sinh \left(\frac{m}{n} \Omega\right)} \\
0 & 0 & 1
\end{array}\right] \cdot\left[\begin{array}{c}
V_{1} \\
I_{1} \\
1
\end{array}\right]}
\end{aligned}
$$

where $\Omega=\sqrt{i \omega R C}$.

If we continue to consider $v_{N O I S E, m, n}$ like a voltage generator, making an equivalence between the scheme in Fig. 11 and the one in Fig. 12, it is straightforward to see that:

$$
\begin{aligned}
& v_{E Q, m}=v_{N O I S E, m, n} \cdot \cosh \left(\frac{m}{n} \Omega\right)=v_{N O I S E, m, n} \cdot H_{\frac{m}{n}}(\omega) \\
& i_{E Q, m}=v_{N O I S E, m, n} \cdot \sqrt{\frac{i \omega C}{R}} \sinh \left(\frac{m}{n} \Omega\right)=v_{N O I S E, m, n} \cdot Y_{\frac{m}{n}}(\omega)
\end{aligned}
$$

where $H_{\frac{m}{n}}(\omega)$ and $Y_{\frac{m}{n}}(\omega)$ are the transfer functions to be used to calculate the equivalent input series and parallel noise generators starting from the $m$-th of $n$ noise generators.

Let's now call $X(\omega)$ the physical quantity (a voltage or a current) on which we want to evaluate the noise contribution $X_{m}(\omega)$ due to $v_{N O I S E, m, n}$. Let's assume that $A(\omega)$ and $B(\omega)$ are the transfer functions from the equivalent voltage and current generators to the desired variable, i.e.

$$
X_{m}(\omega)=v_{E Q, m} \cdot A(\omega)+i_{E Q, m} \cdot B(\omega) .
$$

It is trivial to demonstrate that the power spectral density of noise $P S D_{X}(\omega)$ affecting the quantity $X(\omega)$ due to all the noise generators in the RWDC is

$$
P S D_{X}(\omega)=[A(\omega) \quad B(\omega)] \cdot[S(\omega)] \cdot\left[\begin{array}{l}
A^{*}(\omega) \\
B^{*}(\omega)
\end{array}\right] .
$$

The self- and cross-power spectral density matrix $S$ can be calculated from 41 and 42:

$S(\omega)=\lim _{n \rightarrow \infty} \sum_{m=0}^{n-1} P S D_{m, n}(\omega)\left[\begin{array}{ll}H_{\frac{m}{n}}(\omega) H_{\frac{m}{n}}^{*}(\omega) & H_{\frac{m}{n}}(\omega) Y_{\frac{m}{n}}^{*}(\omega) \\ H_{\frac{m}{n}}^{*}(\omega) Y_{\frac{m}{n}}(\omega) & Y_{\frac{m}{n}}(\omega) Y_{\frac{m}{n}}^{*}(\omega)\end{array}\right]$

where $P S D_{m, n}(\omega)$ is the power spectral density of noise of the $\mathrm{m}$-th noise generator when subdividing the RWDC in $n$ segments. Since the RWDC is divided in segments of the same lenght, all the generators $v_{N O I S E, m}$ have the same PSD equal to $4 k T R / n$. For this reason $P S D_{m, n}(\omega)$ can be rewritten $P S D_{n}(\omega)$ and moved outside the sum. At the same time we transform the sum in an integral, aware of the fact that the error we are committing goes to zero as $n \rightarrow+\infty$.

$S(\omega)=\lim _{n \rightarrow \infty} \frac{4 k T R}{n} \int_{0}^{n}\left[\begin{array}{ll}H_{\frac{m}{n}}(\omega) H_{\frac{m}{n}}^{*}(\omega) & H_{\frac{m}{n}}(\omega) Y_{\frac{m}{n}}^{*}(\omega) \\ H_{\frac{m}{n}}^{*}(\omega) Y_{\frac{m}{n}}(\omega) & Y_{\frac{m}{n}}(\omega) Y_{\frac{m}{n}}^{*}(\omega)\end{array}\right] d m$

We choose $\alpha=m / n$ as independent variable for the integration, with $d m=n \cdot d \alpha$. In this way (44) becomes

$$
S(\omega)=\lim _{n \rightarrow \infty} 4 k T R \int_{0}^{1}\left[\begin{array}{ll}
H_{\alpha}(\omega) H_{\alpha}^{*}(\omega) & H_{\alpha}(\omega) Y_{\alpha}^{*}(\omega) \\
H_{\alpha}^{*}(\omega) Y_{\alpha}(\omega) & Y_{\alpha}(\omega) Y_{\alpha}^{*}(\omega)
\end{array}\right] d \alpha
$$

where the integral is to be considered element-wise and $H_{\alpha}$ and $Y_{\alpha}$ are obtained from (41) with the substitution $\alpha=m / n$. The limit now can collapse since doesn't affect the calculation anymore. These four integrals can be calculated in closed form as follows.

$$
S(\omega)=4 k T R\left[\begin{array}{ll}
S_{v v} & S_{v i} \\
S_{i v} & S_{i i}
\end{array}\right]
$$

The solution of these four integrals can be easily calculated considering that

$$
\sqrt{i \omega R C}=(1+i) \sqrt{\frac{\omega R C}{2}}
$$

and considering the equalities in appendix. The four terms in (47) are:

$$
\begin{gathered}
S_{v v}=\frac{\sin (\phi)+\sinh (\phi)}{2 \phi} \\
S_{v i}=\sqrt{\frac{\omega C}{2 R}}(1-i) \frac{\cosh (\phi)+i \cos (\phi)-(1+i)}{2 \phi} \\
S_{i v}=\sqrt{\frac{\omega C}{2 R}}(1+i) \frac{\cosh (\phi)-i \cos (\phi)-(1-i)}{2 \phi} \\
S_{i i}=\frac{\omega C}{R} \frac{\sinh (\phi)-\sin (\phi)}{2 \phi} .
\end{gathered}
$$


The matrix in (47) contains the self-correlation and crosscorrelation between the two equivalent noise generators in Fig. 12 and can be used, together with (43) to calculate the noise contribution of a RWDC in any circuit [11]. Please notice that, in agreement with the laws reported in literature, the two off-diagonal elements are one the complex conjugate of the other. In the next section we will focus on a particular application case: the usage of the RWDC as feedback resistor in a charge-sensitive preamplifier.

\section{POWER SPECTRAL DENSITY OF NOISE WHEN AN RWDC IS USED AS FEEDBACK RESISTOR OF A CSP}

Let's now focus on the specific case when a RWDC is connected in the feedback network of a Charge-Sensitive Preamplifier (CSP, see Fig. 3). Please refer to the definition of $I_{0}, I_{1}, V_{0}$ and $V_{1}$ given in Fig. 12. The $\mathrm{m}$-th contributes $v_{E Q, m}$ and $i_{E Q, m}$ have been replaced by the total noise generators $v_{E Q}$ and $i_{E Q}$ obtained from the integration on the whole RWDC.

Given the symmetry of the system, let's consider the terminal on the left to be connected to the output of the preamplifier and the right terminal to the input. Being the first a low-impedance voltage source and the second a virtualground node, we can perform the noise propagation analysis considering both RWDC terminals short-circuited to ground, that means $V_{0}=V_{1}=0$. The current $I_{0}$ is the current flowing to the preamplifier output, while the equivalent current noise produced by the RWDC and referred to the preamplifier input is $I_{1}$. Being the preamplifier output a low impedance, we can can assume that the current $i_{E Q}$ flows completely to ground and cannot contribute to $I_{1}$. Only $v_{E Q}$ gives a contribute to $I_{1}$ with amplitude $v_{E Q} / Z_{R W D C}$ where $Z_{R W D C}$ has been defined in (14). The equation in (42) can thus be rewritten as follows to obtain the transfer function between $v_{e q}, i_{e q}$ and the total input noise current $I_{1}$.

$$
\begin{aligned}
& \left.I_{1}\right|_{v_{e q}, i_{e q}}(\omega)=v_{E Q} \cdot A(\omega)+i_{E Q} \cdot B(\omega) \\
& \left.I_{1}\right|_{v_{e q}, i_{e q}}(\omega)=v_{E Q} \cdot-\sqrt{\frac{i \omega C}{R}} \frac{1}{\sinh (\sqrt{i \omega R C})}+i_{E Q} \cdot 0
\end{aligned}
$$

From (53) and (43) we can easily derive the expression of the current PSD of noise flowing from the right terminal of the RWDC into the input node of the CSP.

$$
\begin{aligned}
\operatorname{PSD}_{I_{1}}(\omega) & =4 k T R[A(\omega) \\
& =4 k T R|A(\omega)|^{2} S_{v v}
\end{aligned}
$$

With trivial calculations we can write the total current PSD of noise insisting on the input node of the preamplifier.

$$
P S D_{I_{1}}(\omega)=\frac{4 K T}{R} \sqrt{\omega \frac{R C}{2}} \cdot\left[\frac{\sin \left(2 \sqrt{\omega \frac{R C}{2}}\right)+\sinh \left(2 \sqrt{\omega \frac{R C}{2}}\right)}{\cosh \left(2 \sqrt{\omega \frac{R C}{2}}\right)-\cos \left(2 \sqrt{\omega \frac{R C}{2}}\right)}\right] .
$$

Although (55) can appear to be quite unfamiliar to the reader, its practical interpretation is definitely straightforward. Let's try to analyze its asymptotic behaviour in the low- and highfrequency limits.
For $\omega \rightarrow 0$ the expression 55 clearly goes to

$$
\left.P S D_{I_{1}}\right|_{\omega \rightarrow 0}=\frac{4 k T}{R}
$$

while the high-frequency behaviour is well represented by

$$
\left.P S D_{I_{1}}\right|_{\omega \rightarrow+\infty}=\frac{4 k T}{R} \sqrt{\omega \frac{R C}{2}} .
$$

These two formulas demonstrate that the noise at low frequencies, when the effects of the capacitive coupling to bulk are negligible, is practically white. Above the noise corner frequency, that can be found equating (56) and (57), the PSD raises with the square root of frequency. The noise corner frequency is

$$
f_{\text {CORNER }}=\frac{1}{2 \pi\left(\frac{\mathrm{RC}}{2}\right)} .
$$

The fact that the expression in (55) in the limit for negligible capacitive coupling (i.e. $C \rightarrow 0$ ) tends to $4 k T / R$ is a confirmation of the proposed model's coherence with the noise properties of an ideal resistor.

Let's now compare the closed-form PSD expression with the result obtainable with a circuit simulator. In the latter case the RWDC is simply modeled as chain of $\mathrm{n}$ T-shaped cells, each one made of two $R /(2 n)$ resistors in series with a capacitor $C / n$ to ground in the middle. As can be seen in Fig. 13 the lowfrequency behaviour is modeled correctly already with 4 cells up to the noise corner frequency. Increasing the number of cells doesn't change substantially the low-frequency behaviour but sets the maximum frequency validity of the simulation. In the high-frequency limit, the first capacitor of the chain shunts the resistor to ground, turning it into the only current noise contributor respect to the nearby terminal. In general, this resistor has a value of $\alpha R / n$, with $\alpha$ being a parameter between 0.5 and 1 depending on the elementary cell structure (1/2 in the case of a T-shaped cell). Equating the expressions of the PSD of the first resistor segment

$$
P S D_{\mathrm{FS}}=\frac{4 k T n}{R \alpha}
$$

with the simplified expression of the total PSD of a RWDC for high frequencies (57) we can write the frequency limit of the PSD calculated with a n-cell simulation.

$$
f_{M A X, n}=\left(\frac{n}{\alpha}\right)^{2} f_{C O R N E R}
$$

\section{EXPERIMENTAL VERIFICATION OF THE PROPOSED NOISE MODEL}

A $100 \mathrm{M} \Omega$ integrated polysilicon resistor was realized in AMS C35B4C3 technology [4]. Its dimensions are $350 \mu \mathrm{m}$ by $400 \mu \mathrm{m}$. This resistance was realized with the minimum width allowed by the technology Design Rule-Check (DRC): $0.8 \mu \mathrm{m}$. Due to its geometry, the realized device has thus $10 \mathrm{pF}$ of total distributed capacitance to bulk. The resistor is made of ten $10 \mathrm{M} \Omega$ segments with $1 \mathrm{pF}$ stray capacitance each. Under each segment a n-well guard was realized. For the noise analysis let's just consider all the RWDC wells to be connected to ground. It can be demonstrated that the solution proposed in section IV is efficient for the impedance correction but doesn't 


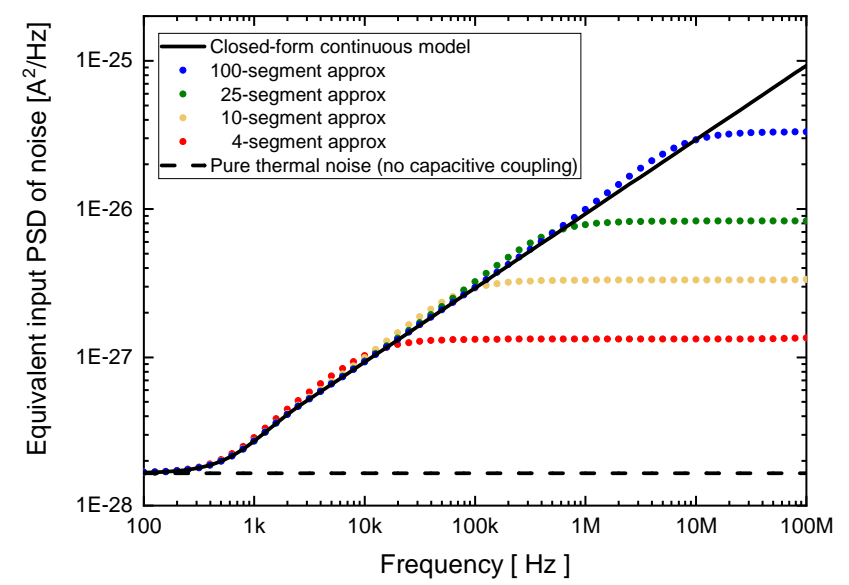

Fig. 13. Parallel current noise produced by an integrated $100 \mathrm{M} \Omega$ feedback resistance with $10 \mathrm{pF}$ of total parasitic capacitance to bulk. Noise SPICE simulations obtained with discrete R-C chain models with different segmentation are compared to the closed-form noise model.

change the noise behaviour of the RWDC. In fact, as long as the impedance of the well-driving circuit is sufficiently low, each n-well can be considered connected to ground.

A low-noise CSP [12] was used to evaluate the total input current PSD of noise generated by the polysilicon RWDC. For the PSD measurements an Agilent 4395A Network Spectrum Analyzer was connected to the CSP output. First of all the intrinsic instrument noise was checked to be considerably lower than the one coming from the CSP at all frequencies in all the possible experimental conditions. The equivalent input PSD was calculated dividing the output PSD by the square modulus of the CSP transfer function. The latter was measured with the same instrument. The measurement was repeated connecting different capacitors $(4 \mathrm{pF}$ and $100 \mathrm{pF})$ to the input of the CSP so as to simulate various detector junction capacitances. The results are reported in Fig. 14. The preamplifier used for this measurement was designed for high-resolution spectroscopy. Its flicker noise contribution is confirmed to be negligible under the experimental conditions of this work.

We thus proceeded quantifying the coherence of the RWDC noise model with the experimental data. The value of the experimental preamplifier input stray capacitance is not known a priori. In the simulation this value was chosen to best fit the experimental data in the $f^{2}$-rise region (above $200 \mathrm{kHz}$ for the $4 \mathrm{pF}$ case and above $40 \mathrm{kHz}$ for the $100 \mathrm{pF}$ case), where the noise effects of the RWDC are not appreciable. In order to make a meaningful comparison it is necessary to assign a reasonable error bar to the experimental data. Unfortunately this is not trivial due to the nature of the measurement and the instrument used. To solve this issue and make a proper $\chi^{2}$ test, the error bars on the experimental data were calculated as follows. The simulation models are clearly valid in the $f^{2}$-rise frequency region. We can thus evaluate the error of the $n$-th experimental point from the root mean square of the relative
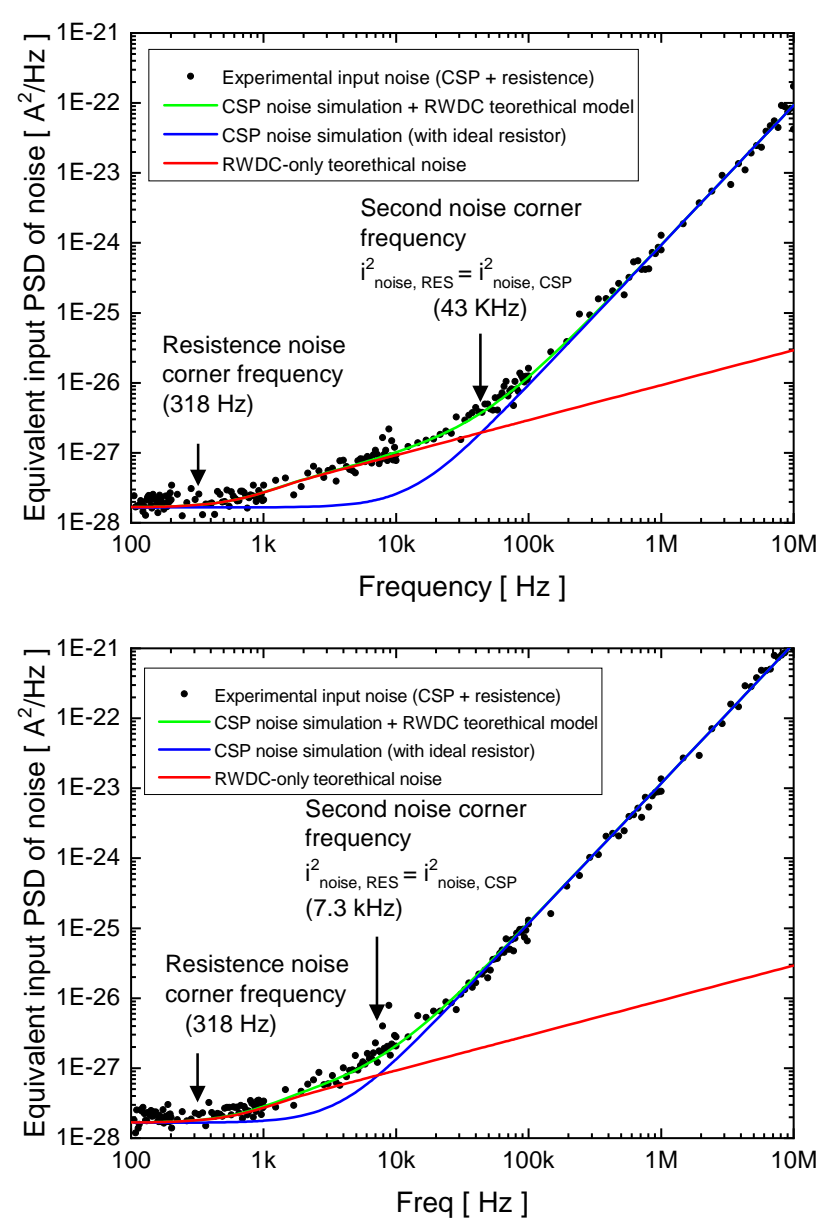

Fig. 14. Input referred current noise of a charge-sensitive preamplifier with the integrated $100 \mathrm{M} \Omega$ resistor as feedback. The input-referred capacitance is $4 \mathrm{pF}$ in the top figure and $100 \mathrm{pF}$ in the bottom figure. Some peaks at $150 \mathrm{~Hz}$ and $200 \mathrm{~Hz}$ were removed from the dataset since generated by the harmonics of the $50 \mathrm{~Hz}$ power supply.

deviation:

$$
\sigma_{n}=x\left(f_{n}\right) \cdot \sqrt{\frac{\sum_{m}\left(\frac{x\left(f_{m}\right)-\exp \left(f_{m}\right)}{x\left(f_{m}\right)}\right)^{2}}{\sum_{m} 1}}
$$

where the sum over $\mathrm{m}$ stands for a sum on the PSD points above the second corner frequency, $x\left(f_{m}\right)$ is the experimental point and $\exp \left(f_{m}\right)$ is the expected value at frequency $f_{m}$. The standard relative deviation averaged between the two dataset of Fig. 14 is equal to $25 \%$. The reduced $\chi^{2}$ calculated on the two experimental PSD up to the second noise corner frequency is equal to 1.05 for $4 \mathrm{pF}$ input capacitance and 1.12 for $100 \mathrm{pF}$. The two associated P-values are respectively $31 \%$ and $18 \%$, both well above the commonly accepted threshold of 5\%, confirming the validity of the proposed model.

The two plots in Fig. 14 highlight the possible relevance of the $\sqrt{f}$ noise rise from a RWDC in some practical situations. Depending on the series input noise of the CSP's opamp and the detector stray capacitance, the non-white component of the RWDC noise can manifestly appear or be partially (or 
completely) masked by the $f^{2}$ (series white noise) and $f$ (series $1 / \mathrm{f}$ noise) opamp contributions.

\section{FINAL CONSIDERATIONS}

In this work the effects induced in high-value integrated resistors by the distributed stray capacitance have been pointed out, both in terms of impedance and noise.

When connecting an RWDC as feedback resistor of a CSP without applying impedance corrections, the net effect can range from distortion of the exponential signal to resonating behaviour. It is possible to perform an impedance correction of an RWDC separating it into several segments and driving the n-wells underlying each segment with proper resistive voltage divider networks. The number of required segments depends on the physical properties of the RWDC and the desired impedance degree of approximation: higher the number of segments, wider the approximation validity bandwidth. Such device would be perfect for the realization of compact, integrated charge-sensitive preamplifiers without external components [13].

The distributed capacitance not only affects the impedance of the RWDC but also its noise properties. When connecting a RWDC to two low-impedance nodes it generates white noise up to a specific corner frequency. Above this frequency the power spectral density of noise rises with the square root of frequency.

Although this work refers mainly to integrated polysilicon resistors, the same considerations apply also to those discrete resistors that have a considerable ratio between stray capacitance to ground and resistance. Even the channel of long MOS transistors used in low-current active transconductors (characterized by high gate-channel capacitance and mediumto-high linear resistivity) may suffer from the same issues, as long as the resistance-capacitance product brings the $\sqrt{f}$ noise corner frequency down into the operating bandwidth of the circuit.

Further works will consider the effects of different types of spectroscopic shaping filters on the $\sqrt{f}$ noise. Resolution degradation, optimal filtering and other practical aspects will be pointed out.

\section{APPENDIX}

The solution of (46) can be calculated considering the following four equalities:

$$
\begin{aligned}
& \cosh \left[\alpha(1+i) \sqrt{\frac{\omega R C}{2}}\right] \cdot \cosh \left[\alpha(1-i) \sqrt{\frac{\omega R C}{2}}\right]= \\
& \frac{1}{2}[\cos (\alpha \sqrt{2 \omega R C})+\cosh (\alpha \sqrt{2 \omega R C})], \\
& \cosh \left[\alpha(1+i) \sqrt{\frac{\omega R C}{2}}\right] \cdot \sinh \left[\alpha(1-i) \sqrt{\frac{\omega R C}{2}}\right]= \\
& -\frac{i}{2}[\sin (\alpha \sqrt{2 \omega R C})+i \sinh (\alpha \sqrt{2 \omega R C})],
\end{aligned}
$$

$$
\begin{aligned}
& \cosh \left[\alpha(1-i) \sqrt{\frac{\omega R C}{2}}\right] \cdot \sinh \left[\alpha(1+i) \sqrt{\frac{\omega R C}{2}}\right]= \\
& \frac{i}{2}[\sin (\alpha \sqrt{2 \omega R C})-i \sinh (\alpha \sqrt{2 \omega R C})] \\
& \sinh \left[\alpha(1+i) \sqrt{\frac{\omega R C}{2}}\right] \cdot \sinh \left[\alpha(1-i) \sqrt{\frac{\omega R C}{2}}\right]= \\
& \frac{1}{2}[\cosh (\alpha \sqrt{2 \omega R C})-\cos (\alpha \sqrt{2 \omega R C})] .
\end{aligned}
$$

\section{REFERENCES}

[1] A. Georgiev and W. Gast, "Digital pulse processing in high resolution, high throughput, gamma-ray spectroscopy," IEEE Transactions on $\mathrm{Nu}$ clear Science, vol. 40, no. 4, pp. 770-779, Aug 1993.

[2] A. Pullia and S. Capra, "Design of a resistorless ASIC preamplifier for hpge detectors with non-linear pole/zero cancellation and controlled fastreset feature," IEEE Nuclear Science Symposium Conference Record, pp. 86-90, 2012.

[3] N. D. Marco, "Searching for neutrinoless double-beta decay with gerda," Nuclear Instruments and Methods in Physics Research Section A: Accelerators, Spectrometers, Detectors and Associated Equipment, 2019.

[4] "Ams home page:." [Online]. Available: https://ams.com

[5] A. Spessot, M. Molteni, D. Ventrice, and P. Fantini, "A physics-based compact model for polysilicon resistors," IEEE Electron Device Letters, vol. 31, no. 11, pp. 1251-1253, Nov 2010.

[6] E. Weisstein, "Puiseux series," from MathWorld - A Wolfram Web Resource. [Online]. Available: http://mathworld.wolfram.com/PuiseuxSeries.html

[7] C. Siegel, Topics in Complex Function Theory: Elliptic functions and uniformization theory, ser. Interscience tracts in pure and applied mathematics. Wiley-Interscience, 1973, pp. 98-99.

[8] S. Capra and A. Pullia, "Study of the effects of parasitic capacitance on large integrated feedback resistors for charge-sensitive preamplifiers," 2014 IEEE Nuclear Science Symposium Conference Record, 2016.

[9] Z. Zhou and P. Warr, "A segmentation layout guarding technique to mitigate parasitic capacitance of integrated resistors," Analog Integrated Circuits and Signal Processing, vol. 93, no. 2, pp. 237-243, Nov 2017.

[10] C.-C. Chen, J. Ke, and Y.-T. Chen, "New method of state-space formulation for degenerate circuit and coupling circuit," Mathematical Problems in Engineering, vol. 2019, 2019.

[11] G. Vasilescu, Electronic Noise and Interfering Signals. Springer, Berlin, Heidelberg, pp. 39-41, Section 2.3.5.

[12] S. Capra, D. Mengoni, J. Dueñas, P. John, A. Gadea, R. Aliaga, J. Dormard, M. Assie, and A. Pullia, "Performance of the new integrated front-end electronics of the trace array commissioned with an early silicon detector prototype," Nuclear Instruments and Methods in Physics Research, Section A: Accelerators, Spectrometers, Detectors and Associated Equipment, vol. 935, pp. 178-184, 2019.

[13] A. Pullia, E. Frontini, and S. Capra, "Design of an ultra-fast low-noise charge-sensitive microprobe for semiconductor detectors," 2014 IEEE Nuclear Science Symposium Conference Record, 2016. 